\title{
The different dimensions of nanotechnology
}

\author{
Materials can have one, two or three dimensions in the nanoscale regime, which adds to the variety of \\ phenomena that can be explored in nanoscience and technology.
}

Many definitions of nanotechnology refer to dimensions: according to the National Nanotechnology Initiative (NNI) in the United States, for instance, "nanotechnology is the understanding and control of matter at dimensions between approximately 1 and 100 nanometres, where unique phenomena enable novel applications." (ref. 1). Sometimes just one or two dimensions are in the nanoregime, as in quantum wells and nanowires respectively, and sometimes all three dimensions are nanoscale, as in quantum dots and nanocrystals. Sometimes the challenge facing researchers is to make every dimension as small as possible, as in nanoelectronic devices, but other times the aim is to make at least one dimension as large as possible, as in fibres based on carbon nanotubes. Often the challenges lie in other directions, such as reducing cost, scaling-up production or working in environments other than the low-temperature and ultrahigh-vacuum conditions favoured by many physicists.

Carbon nanotubes are the ultimate one-dimensional material - which means, perhaps confusingly, that they have two nanoscale dimensions. Almost two decades after their discovery, nanotubes remain the subject of countless research papers. Some of these papers are about condensed-matter physics of the most fundamental kind, such as quantum phase transitions (see p147 and ref. 2), and offer the opportunity to explore ideas first proposed by theorists decades ago, whereas other papers address problems related to the use of nanotubes in various forms of electronics, such as nanotubebased semiconducting inks for printed electronics (see p143 and ref. 3), and involve a multidisciplinary mix of physics, chemistry, materials science and more. Although nanotube-based electronic devices have yet to reach the market, there are high hopes for other, less exotic, applications $^{4}$ - especially as production costs come down. According to Frost and Sullivan ${ }^{5}$, the production costs of multiwalled carbon nanotubes are expected to fall from $\$ 100$ per kilogram at present to about $\$ 10-20$ per kilogram in a few years; the company cite flame-retardant materials - sales of which amount to almost $\$ 3$ billion per year - as an example of the sort of market that will open up for nanotubes as production costs fall.

\section{Of course three dimensions of space are not enough for some physicists and cosmologists.}

Moving into two dimensions, graphene is the hottest nanomaterial today, and like its rolled-up relative, the single-walled carbon nanotube, these single sheets of carbon atoms are also the subject of many research papers (and editorials in this journal ${ }^{6,7}$ ). As with nanotubes, some of these papers are about fundamental issues in physics that date back to famous names such as Klein and Landau. And again like nanotubes, graphene displays remarkable electronic and structural properties, which means that a large number of groups are tackling the challenges of exploiting these properties in next-generation nanoscale devices. Moreover, many of the experimental techniques developed to study nanotubes can also be used on graphene, which helps explain why so much progress has been made in the past five years.

Staying in two dimensions, on page 167 Hari Manoharan and coworkers at Stanford describe a technique called quantum holography that allows information to be stored in a twodimensional electron gas on a metal surface. It might be thought that using a scanning tunnelling microscope (STM) to position individual atoms or molecules on a surface would represent the ultimate in information storage, but the Stanford team betters this limit by moving into a third dimension - energy. Manoharan and co-workers show that carbon monoxide molecules can be placed on a copper surface by a STM, such that information is stored in the two-dimensional electron gas confined by the molecules, rather than by the molecules themselves. By changing the voltage applied to the microscope, it is possible to read the information that is stored in the electron gas at different energies. Nobody is going to be massproducing devices based on this approach, as Eric Heller points out on page 141, but it is still a remarkable piece of physics.

Of course three dimensions of space are not enough for some physicists and cosmologists, who prefer to believe that the universe has nine or ten spatial dimensions, and that particles are strings rather than point-like objects. Traditionally, the extra dimensions found in string theories have been many orders of magnitude smaller than the nanoscale, although some theorists have argued that some of these extra dimensions might be 'large' (of the order of a millimetre in some theories $)^{8}$. Whatever the size of these possible extra dimensions, the cutting edge nanoscale circuitry and devices found in detectors at a variety of telescopes and particle accelerators are ready to find them - if they exist.

\footnotetext{
References

1. <http://www.nano.gov/html/facts/whatIsNano.html>

2. Deshpande, V. V. et al. Science 323, 106-110 (2009).

3. Kanungo, M., Lu, H., Malliaras, G. G. \& Blanchet, G. B. Science 323, 234-237 (2009).

4. Nature Nanotech. 4, 1 (2009)

5. <http://www.frost.com/prod/servlet/press-release pag?docid $=159277406>(18$ February 2009)

6. Nature Nanotech. 2, 191 (2007).

7. Nature Nanotech. 3, 517 (2008).

8. Adelberger, E., Heckel, B. \& Hoyle, C. D. Phys. World 18, 41-46 (April 2005).
} 